\title{
Peritoneal bladder fistula following radiotherapy for cervical cancer: A case report
}

\author{
FAN SHI, TAO WANG, JIQUAN WANG, BEINA HUI, YANLAN CHAI, JUAN WANG and ZI LIU \\ Department of Radiation Oncology, First Affiliated Hospital of Xi'an Jiaotong University, Xi'an, Shaanxi 710061, P.R. China
}

Received March 31, 2015; Accepted May 12, 2016

DOI: $10.3892 / \mathrm{ol} .2016 .4820$

\begin{abstract}
The occurrence of a peritoneal bladder fistula as a result of radiation cystitis following radiotherapy for cervical cancer is extremely rare and, to the best of our knowledge, has not been reported previously. The present study reports the case of a 50-year-old woman who was diagnosed with cervical cancer 20 years previously and was treated with radiotherapy. The patient was diagnosed with radiation cystitis 10 years ago, which was treated with Chinese medicine, and began experiencing sudden abdominal pain and bowel difficulties following urination 3 years ago. B-ultrasound examination at The People's Hospital of Tongchuan (Tongchuan, China) detected the presence of abdominal pelvic fluid. Following antibiotic (levofloxacin for 5 days) and ascites extraction treatment, symptoms were relieved without recurrence. However, 5 days prior to admission to the First Affiliated Hospital of Xi'an Jiatong University (Xi'an, China) on June 25, 2014, the patient experienced difficulty when urinating, abdominal pain and bloating, but did not experience frequent urination, hematuria or fever. Cystoscopic examination revealed a visible fistula on the bladder wall measuring $1 \times 1 \mathrm{~cm}$ in diameter. Cytoscopic examination 1 month after catheterization and ascites extraction revealed no evidence of the fistula. The patient was followed up every 3 months for a year and a half, and is currently alive and well. In conclusion, the occurrence of peritoneal bladder fistula following radiation therapy is rare and cystoscopy is the preferred method of examination and diagnosis. Early detection and treatment may significantly improve the prognosis of patients.
\end{abstract}

\section{Introduction}

Radiation cystitis frequently occurs following radiotherapy treatment for cervical, colorectal and prostate cancer, in addition to other pelvic tumors, with an incidence rate of $\leq 5 \%$ of all

Correspondence to: Dr Zi Liu, Department of Radiation Oncology, First Affiliated Hospital of Xi'an Jiaotong University, 277 Yanta West Road, Xi'an, Shaanxi 710061, P.R. China

E-mail: liuzmail@163.com

Key words: intraperitoneal bladder fistula, radiotherapy, cervical cancer patients that undergo radiotherapy (1). Previous studies have reported that acute radiation cystitis, which typically develops 2-4 weeks after radiotherapy with a severity of <II [Radiation Therapy Oncology Group staging system (2)], occurs in $21-25 \%$ of all patients that undergo radiotherapy, while chronic radiation cystitis has an incidence rate of $1 \%$ (3). The occurrence and severity of radiation cystitis has gradually decreased due to technical advances in the field of radiotherapy (4). To the best of our knowledge, the occurrence of a peritoneal bladder fistula as a result of radiation cystitis following radiotherapy for cervical cancer has not been reported in the literature. The present study reports a case of a peritoneal bladder fistula, which occurred 20 years after the administration of radiotherapy for cervical cancer, and discusses the possible causes and potential treatments for this condition.

\section{Case report}

A 50-year-old woman, who was previously diagnosed with cervical cancer and treated with radiotherapy 20 years ago in June 1994, was admitted to the First Affiliated Hospital of Xi'an Jiatong University (Xi'an, China). No recurrence or metastasis was observed during the periodic examinations that followed the diagnosis. The patient began experiencing intermittent increased urination frequency, urination urgency and hematuria 10 years ago, and was subsequently treated with Chinese medicine for 1 year, which alleviated the symptoms. However, 3 years ago, the patient experienced sudden abdominal pain and bowel difficulties following urination. B-ultrasound examination at The People's Hospital of Tongchuan (Tongchuan, China) detected the presence of abdominal pelvic fluid. Following antibiotic (levofloxacin for 5 days) and ascites extraction treatment, symptoms were relieved without any recurrence. Five days prior to admission to the First Affiliated Hospital of Xi'an Jiatong University on June 25, 2014, the patient experienced difficulty urinating, abdominal pain and bloating, but did not experience frequent urination, hematuria or fever. B-ultrasound examination indicated a rough bladder wall and a large pelvic effusion. Laboratory examinations performed 5 days prior to admission revealed mild urinary occult blood, severe urinary protein, blood urea nitrogen (BUN) levels of $12.34 \mathrm{mmol} / \mathrm{l}$ and creatinine (CREA) levels of $268 \mathrm{umol} / \mathrm{l}$. Further examinations revealed that blood pressure, heart rate, temperature and chest X-ray were all normal, in addition to abdominal tension, no tenderness or rebound 
Table I. Patient BUN and CREA levels in the blood, urine and ascites following hospital admission.

\begin{tabular}{lccc}
\hline & $\begin{array}{c}\text { Blood } \\
\text { (normal range) }\end{array}$ & Urine & Ascites \\
\hline BUN, mmol/l & $18.53(2.60-7.50)$ & 72.24 & 31.05 \\
CREA, mmol/1 & $288(41.00-73.00)$ & 12124 & 1371 \\
\hline
\end{tabular}

BUN, blood urea nitrogen; CREA, creatinine.

Table II. Examination results for ascites and urine.

\begin{tabular}{lcc}
\hline Parameters & Ascites & Urine \\
\hline Color & Light yellow & Light yellow \\
Transparency & Transparent & Transparent \\
Clot & No clot & No clot \\
Rivalta test & Negative & Negative \\
Total cells & $72 \times 10^{6} / 1$ & $21 \times 10^{6} / 1$ \\
White blood cells & $38 \times 10^{6} / 1$ & $11 \times 10^{6} / 1$ \\
\hline
\end{tabular}

Table III. Biochemical test results for ascites and urine.

\begin{tabular}{llc}
\hline $\begin{array}{l}\text { Biochemical } \\
\text { parameters }\end{array}$ & \multicolumn{1}{c}{ Ascites } & Urine \\
\hline $\begin{array}{l}\text { Total protein, g/l } \\
\text { Glucose, mmol/1 }\end{array}$ & $7.53(<40)$ & $3.02(60-80)$ \\
$\begin{array}{l}\text { Lactate } \\
\text { dehydrogenase, U/1 }\end{array}$ & $40.6(109-245)$ & $32.2(109-245)$ \\
$\begin{array}{l}\text { Chlorine, mmol/1 } \\
\text { Adenosine }\end{array}$ & $96.9(96-108)$ & $35.6(96-108)$ \\
deaminase, $\mathrm{U} / \mathrm{l}$ & $0.7(<20)$ & $0(<20)$ \\
\hline
\end{tabular}

tenderness, no liver or spleen palpation, no percussion pain in the kidney area, positive shifting dullness and normal bowel sounds. Gynecological examination was normal. The diagnosis at admission was suspected as i) ascites of unknown reason and ii) cervical cancer. Routine blood and liver function tests following admission were normal, and tumor marker levels, including cancer antigen (CA)125, CA153, CA199, carcinoembryonic antigen and squamous cell carcinoma antigen, were also normal. Chest and abdominal computed tomography and pelvic magnetic resonance imaging examination excluded tumor recurrence and metastasis. BUN and CREA levels in ascites, blood and urine were tested following hospital admission and are presented in Table I. Routine biochemical tests were performed for ascites and urine, which indicated that the ascitic fluid was transudate (Tables II and III). Pathological examination of ascites revealed the presence of mesothelial cells and lymphocytes; however, no malignant tumor cells were identified. Therefore, a peritoneal bladder fistula was suspected and urine ascites were subsequently obtained. Cystoscopic examination revealed pale and thick bladder mucosa,

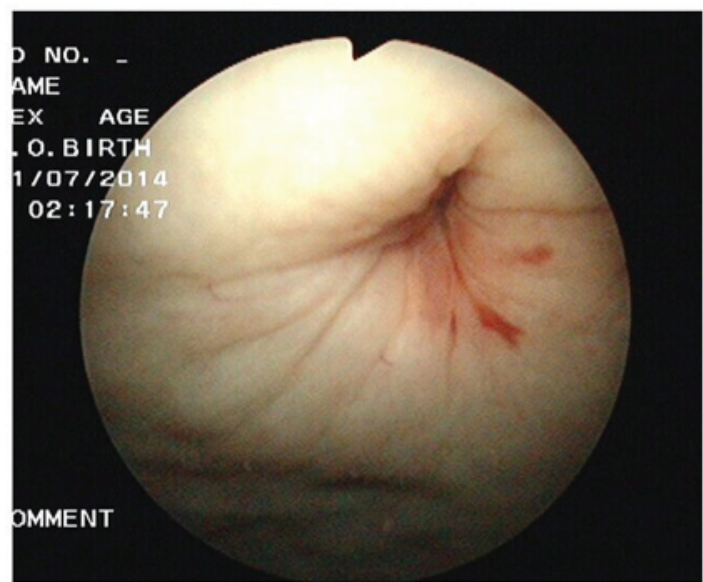

Figure 1. Cystoscopic examination of the bladder. Examination revealed pale and thick bladder mucosa, vascular texture thickening and a fistula (diameter, $1 \times 1 \mathrm{~cm}$ ) on the bladder wall.

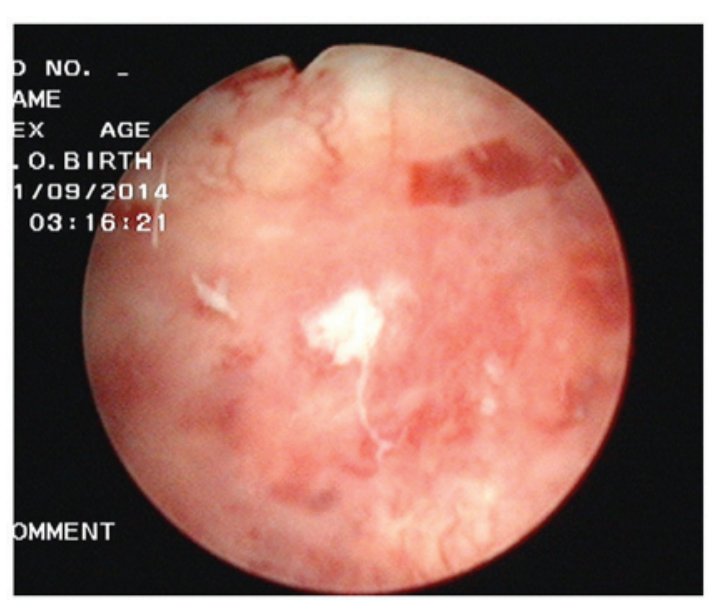

Figure 2. Cystoscopic examination of the bladder 1 month later. No evidence of the fistula is visible.

vascular thickening and the formation of a visible fistula on the bladder wall measuring 1x1 cm in diameter (Fig. 1). The preliminary diagnosis was the presence of a peritoneal bladder fistula with radiation cystitis. Following catheterization and ascites extraction, the symptoms were relieved and the blood BUN and CREA levels had reduced to $6.4 \mathrm{mmol} / \mathrm{l}$ and $166 \mathrm{umol} / \mathrm{l}$, respectively. Cytoscopic examination 1 month later revealed no evidence of the fistula (Fig. 2). The final diagnosis was confirmed as a peritoneal bladder fistula as a result of radiotherapy for cervical cancer. The patient was followed up everyday 3 months for a year and a half, and is currently alive and well.

\section{Discussion}

Radiation cystitis typically affects the trigone of the urinary bladder and the surrounding tissues (5). Generally, the tolerated radiation dose of the bladder is considered to be $60 \mathrm{~Gy}$; therefore, radiation cystitis may occur if the dose exceeds this. A previous study reported that radiation cystitis develops after receiving a dose that is $10 \%$ higher than the regular dose (60 Gy) (6,7). The primary pathological symptoms of radiation 
cystitis include ulcers accompanied by bleeding, inflammatory cell infiltration and epithelial cell atrophy or hyperplasia (8). Biopsies of bladder tissues exhibiting radiation cystitis usually demonstrate shedding of bladder transitional epithelial cells and cellulose inflammatory exudates. Necrotic tissue below the epithelium is also often observed, in addition to a thin layer of granulation containing numerous thin-walled blood vessels and fibrous tissue hyperplasia with hyaline degeneration, which involves the muscular layer and the outer membrane (9). Shedding of bladder epithelial mucosal cells results in the loss of epithelial protection and the permeability barrier (9). Consequently, extensive amounts of water and urea permeate into the smooth muscle layer of the bladder, resulting in nerve hyperexcitability, detrusor muscle dysfunction and sudden, painless hematuria associated with bladder irritation (9).

Radiation cystitis may be divided into three types according to the time of occurrence and severity: Acute radiation cystitis, chronic radiation cystitis and radiation bladder fistula (10). Acute radiation cystitis usually occurs within 6 months of radiotherapy and is characterized by gross or microscopic hematuria associated with urinary tract irritation symptoms, including increased urinary frequency and urgency. These symptoms may be relieved when radiotherapy is terminated or following relevant treatment (11). Chronic radiation cystitis accounts for $80 \%$ of cases of radiation cystitis and usually occurs $2-4$ years after radiotherapy due to the delayed healing of acute radiation cystitis or late stage radiation injuries (11). Radiation bladder fistulas present further development of chronic radiation cystitis. Obstetric fistulas are the most common, while peritoneal bladder fistulas are rare (12). Radiation bladder fistulas are associated with the distribution of the radiation dose (5). The trigone of the urinary bladder receives the highest radiation doses during pelvic radiotherapy; therefore, radiation cystitis and obstetric fistulas usually occur in this region (5). To the best of our knowledge, no cases of peritoneal bladder fistulas as a result of radiotherapy have been reported previously. The primary causes of bladder fistulas are trauma, surgery, tumors and radiotherapy, and surgery is the standard treatment option (13).

In the present study, a peritoneal bladder fistula occurred as a result of radiotherapy, bladder cystitis and excessive urinary holding. Long-term radiation bladder cystitis leads to fibrosis of bladder mucosa and a reduction in bladder wall tension. Therefore, sudden increases in bladder capacity and wall tension may result in rupture. Notably, the rupture observed in the patient of the present study was not located in the trigone of the urinary bladder and was instead located in the back wall of the bladder, thus resulting in the development of a peritoneal bladder fistula and leakage of urine into the peritoneal cavity. In the early stages of the disease, catheterization and other symptomatic treatments, including hemostasis, anti-inflammatory and sodium hyaluronate treatment, may heal the fistula via bladder self-repair (13). However, in patients with delayed healing, long-term stimulation of the peritoneum by urine may result in abdominal pain and infection, and life-threatening infectious peritonitis. The present case demonstrates that urinary holding must be avoided in patients with a history of pelvic radiotherapy as it may lead to the formation of a bladder fistula. In addition, patients with sudden urinary difficulties and ascites following radiotherapy must be monitored for the development of peritoneal bladder fistulas. Cystoscopy is the preferred method of examination and diagnosis, and early detection and treatment may significantly improve the patient prognosis.

In conclusion, the occurrence of peritoneal bladder fistulas following radiation therapy is rare and may easily be misdiagnosed as peritoneal metastasis with ascites. If a patient that has undergone abdominal or pelvic radiotherapy and been diagnosed with chronic radiation cystitis experiences sudden abdominal pain, severe unexplained ascites and dysuria, the possibility of a peritoneal bladder fistula should be considered. Early cystoscopic examination and timely treatment may significantly improve the prognosis.

\section{References}

1. Liem X, Saad F and Delouya G: A practical approach to the management of radiation induced hemorrhagic cystitis. Drugs 75 : 1471-1482, 2015.

2. Cox JD, Stetz J and Pajak TK: Toxicity criteria of the Radiation Therapy Oncology Group (RTOG) and the European Organization for Research and Treatment of Cancer (EORTC). Int J Radiat Oncol Biol Phys 31: 1341-1346, 1995.

3. Coman JM, Mcclure D, Pritchett R, Kozlowski P and Hampson NB: Treatment of radiation induced hemorrhagic cystitis with hyperbaric oxygen. J Urol 169: 2200-2202, 2003.

4. Wit EM and Horenblas S: Urological complications after treatment of cervical cancer. Nat Rev Urol 11: 110-117, 2014.

5. Pourquier H, Delard R, Achille E, Daly NJ, Horiot JC, Keiling R, Pigneux J, Rozan R, Schraub S and Vrousos C: A quantified approach to the analysis and prevention of urinary complications in radiotherapeutic treatment of cancer of the cervix. A quantified approach to the analysis and prevention of urinary complications in radiotherapeutic treatment of cancer of the cervix 13: 1025$1033,1987$.

6. Appelt AL,Bentzen SM,Jakobsen A and Vogelius IR: Dose-response of acute urinary toxicity of long course preoperative chemoradiotherapy for rectal cancer. Acta Oncol 54: 179-186, 2015.

7. Rosewall T, Catton C, Currie G, Bayley A, Chung P, Wheat J and Milosevic M: The relationship between external beam radiotherapy dose and chronic urinary dysfunction - a methodological critique. Radiother Oncol 97: 40-47, 2010.

8. Kanai A, Epperly M, Pearce L, Birder L, Zeidel M, Meyers S, Greenberger J, de Groat W, Apodaca G and Peterson J: Differing roles of mitochondrial nitric oxide synthase in cardiomyocytes and urothelial cells. Am J Physiol Heart Circ Physiol 286: H13-H21, 2004.

9. Li A, Sun J and Chao H: Late bladder complications following radiotherapy of carcinoma of the uterine cervix. Zhonghua Fu Chan Ke Za Zhi 30: 741-743, 1995 (In Chinese).

10. Brzozowska A, Idziak M, Burdan F and Mazurkiewicz M: Symptoms and treatment of radiation-induced reactions. Pol Merkur Lekarski 38: 283-287, 2015 (In Polish).

11. Tasdemir N, Abali R, Celik C, Yazici CM and Akkus D: The use of an anterior abdominal wall peritoneal flap in the laparoscopic repair of vesicouterine fistula. Int Surg 99: 673-676, 2014.

12. Iloabachie GC and Njoku O: Vesico-uterine fistula. Br J Urol 57: 438-439, 1985.

13. Chuang YC, Kim DK, Chiang PH and Chancellor MB: Bladder botulinum toxin $\mathrm{A}$ injection can benefit patients with radiation and chemical cystitis. BJU Int 102: 704-706, 2008. 